\title{
Synchronization of two non-identical coupled exciters in a non-resonant vibrating system of linear motion. Part II: Numeric analysis
}

\author{
Chunyu Zhao ${ }^{\mathrm{a}, *}$, Hongtao Zhu ${ }^{\mathrm{b}}$, Tianju Bai ${ }^{\mathrm{a}}$ and Bangchun Wen ${ }^{\mathrm{a}}$ \\ a School of Mechanical Engineering and Automation, Northeastern University, Shenyang 110004, China \\ ${ }^{\mathrm{b}}$ Faculty of Engineering, University of Wollongong, Wollongong, NSW 2522, Australia
}

Received 19 February 2008

Revised 2008

\begin{abstract}
The paper focuses on the quantitative analysis of the coupling dynamic characteristics of two non-identical exciters in a non-resonant vibrating system. The load torque of each motor consists of three items, including the torque of sine effect of phase angles, that of coupling sine effect and that of coupling cosine effect. The torque of frequency capture results from the torque of coupling cosine effect, which is equal to the product of the coupling kinetic energy, the coefficient of coupling cosine effect, and the sine of phase difference of two exciters. The motions of the system excited by two exciters in the same direction make phase difference close to $\pi$ and that in opposite directions makes phase difference close to 0 . Numerical results show that synchronous operation is stable when the dimensionless relative moments of inertia of two exciters are greater than zero and four times of their product is greater than the square of their coefficient of coupling cosine effect. The stability of the synchronous operation is only dependent on the structural parameters of the system, such as the mass ratios of two exciters to the vibrating system, and the ratio of the distance between an exciter and the centroid of the system to the equivalent radius of the system about its centroid.
\end{abstract}

Keywords: Self-synchronization, vibration system, frequency capture, vibratory synchronous transmission, stability

\section{Introduction}

In the first part of the present investigation [23], an analytical approach was proposed to study the feature of frequency capture for two non-identical coupled exciters in a non-resonant vibrating system of linear motion, which converts the problem of frequency capture and synchronization of two exciters into that of existence and stability of zero solution of Equations of Frequency Capture. The moment of inertia of each exciter in the Equations of Frequency Capture reduces and there is a coupling moment of inertia between two exciters. This phenomenon has a significant effect on the stability of synchronous operation of two motors. Other researchers had found that the dynamic stability of a system with multi-rigid bodies depends on the inertia coupling in the system. Ye et al. [9] studied the dynamic coupling characteristics of 6-DOF parallel robots and found that there are strong couplings among each channel, which affect the load inertia acting on them. Thus, it was difficult to control 6-DOF parallel robots. In the investigation of the control of two pendulums coupled by a spring, Fradkov and Andrievsky [8] confirmed that the perturbations stemming from both the inertia of the coupling link and that of the motor can provide different influences upon the perturbed system behavior. To our knowledge, no attempt has yet been made to discuss the characteristics of inertia coupling for the mechanical systems with multi-rigid bodies. It is fundamentally

\footnotetext{
*Corresponding author. E-mail: chyzhao@mail.neu.edu.cn.
} 
important to investigate the characteristics of inertia couplings, which can provide the theories for the structural design and control of mechanical systems with multi-rigid bodies.

This paper is aimed at the quantitative analysis of the coupling characteristics of the considered system by virtue of numerical method. The coupling characteristics of the exciters are categorized into load coupling and inertia coupling. The load coupling has an effect on the position of the steady-state operation, i.e., the values of $\omega_{\mathrm{m} 0}^{*}$ and $\alpha^{*}$; the inertia coupling has an effect on the synchronization stability of two motors. As mentioned in the first paper [23], one half of product of the torque of frequency capture and the sine of phase difference between two exciters acts on the motor (leading phase) as load torque to decrease its angular velocity, and the other half acts on another motor (lagging phase) as driving torque to increase its angular velocity. This phenomenon demonstrates that the torque of frequency capture transmits the electromagnetic torque from one motor (with the bigger electromagnetic torque) to the other motor in the system. In particular, after the synchronous rotation of two motors at the steady-state, the power supply of one motor is cut off, i.e., the power source is only supplied to one motor. However, the synchronous rotation of two motors can continue by re-adjusting phase difference between two exciters if the torque of frequency capture is big enough to transmit the driving torque from the motor (with power supply) to another (without power supply) to overcome its load torque. This specific synchronous rotation using only one motor's power supply is called vibratory synchronization transmission [18,20-22].

The rest of this paper is organized as follows: Section 2 analyzes the coupling characteristics of two exciters and Section 3 describes the numerical method used in this paper. In Section 4, we discuss the numeric results of the coupling characteristics of two exciters and Section 5 shows our conclusions of this paper.

\section{Analysis of coupling characteristics of two exciters}

The coupling characteristics of two exciters in a vibrating system are referred to as the characteristic of the load and inertia coupling of two exciters. When two motors operate synchronously at the steady-state, i.e., $\bar{\varepsilon}_{1}=\bar{\varepsilon}_{2}=0$, the load torques of two motors can be respectively rewritten as follows [23]

$$
\begin{aligned}
& \bar{T}_{\mathrm{L} 1}=\frac{1}{2} m_{1} r^{2} \omega_{\mathrm{m} 0}^{2} \cdot m_{1} W_{\mathrm{s} 0}+\frac{1}{2} m_{\mathrm{c}} r^{2} \omega_{\mathrm{m} 0}^{2} \cdot m_{\mathrm{c}} W_{\mathrm{s}} \cos 2 \alpha^{*}+\frac{1}{2} m_{\mathrm{c}} r^{2} \omega_{\mathrm{m} 0}^{2} \cdot m_{\mathrm{c}} W_{\mathrm{c}} \sin 2 \alpha^{*} \\
& \bar{T}_{\mathrm{L} 2}=\frac{1}{2} m_{2} r^{2} \omega_{\mathrm{m} 0}^{2} \cdot m_{2} W_{\mathrm{s} 0}+\frac{1}{2} m_{\mathrm{c}} r^{2} \omega_{\mathrm{m} 0}^{2} \cdot m_{\mathrm{c}} W_{\mathrm{s}} \cos 2 \alpha^{*}-\frac{1}{2} m_{\mathrm{c}} r^{2} \omega_{\mathrm{m} 0}^{2} \cdot m_{\mathrm{c}} W_{\mathrm{c}} \sin 2 \alpha^{*}
\end{aligned}
$$

where $m_{\mathrm{c}}=\sqrt{m_{1} m_{2}}$ is the coupling mass of two exciters; $m_{\mathrm{c}} r^{2} \omega_{\mathrm{m} 0}^{2} / 2$ the coupling kinetic energy of two exciters. Note: in order to be convenient for the analysis, $m_{0}$ and $m_{0} \eta$ are replaced by $m_{1}$ and $m_{2}$ in Eq. (1), respectively.

The coupling inertia of two exciters influences the stability of synchronous operation in a vibrating system. When the items relative to $\sin \gamma_{x}, \sin \gamma_{y}$ and $\sin \gamma_{\psi}$ in the coefficients of $\dot{\bar{\varepsilon}}_{1}$ and $\dot{\bar{\varepsilon}}_{2}$ are considered in integrating the differential equations of motion of two exciters, the denominator of coefficients of the characteristic equation can be rewritten as [23]

$$
E=\omega_{\mathrm{m} 0}^{* 2}\left(4 J_{01}^{\prime} J_{02}^{\prime}+m_{\mathrm{c}}^{2} r^{4} \cdot m_{\mathrm{c}}^{2} W_{\mathrm{s}}^{2} \sin ^{2} 2 \alpha^{*}-m_{\mathrm{c}}^{2} r^{4} \cdot m_{\mathrm{c}}^{2} W_{\mathrm{c}}^{2} \cos ^{2} 2 \alpha^{*}\right)
$$

and

$$
\begin{aligned}
& J_{01}^{\prime}=m_{1} r^{2}\left(1-m_{1} W_{\mathrm{c} 0} / 2\right) \\
& J_{02}^{\prime}=m_{2} r^{2}\left(1-m_{2} W_{\mathrm{c} 0} / 2\right)
\end{aligned}
$$

During the operation of the vibrating system, exciters 1 and 2 respectively excite the motions of the system in $x-, y-$ and $\psi$-directions at the same time, and the vibrations of the system are the linear superposition of these motions. Because two exciters move with the vibrating system, the motions of the system have an effect on the load torques and the moments of inertia of two exciters, as shown in Eqs (1), (2) and (3). In these equations, $m_{1} W_{\mathrm{c} 0}$ and $m_{1} W_{\mathrm{s} 0}$ represent the effect of motions excited by exciter 1 on exciter 1 , and $m_{2} W_{\mathrm{c} 0}$ and $m_{2} W_{s 0}$ represent the effect of motions excited by exciter 2 on exciter 2 . While $m_{\mathrm{c}} W_{\mathrm{s}}$ and $m_{\mathrm{c}} W_{\mathrm{c}}$ represent the effect of motions excited by one exciter on the other exciter; hence they are the parameters that describe the coupling characteristic of two exciters. $W_{\mathrm{s} 0}$ and $W_{\mathrm{c} 0}$ are relative to the sine and cosine of the phase angles in three directions of the vibration, 
respectively; and each item in three directions is positive. $W_{\mathrm{s}}$ and $W_{\mathrm{c}}$ are also relative to the sine and cosine of the phase angles, respectively. However, the item in $W_{\mathrm{s}}$ is positive in $y$-direction (the exciting forces of the two exciters are in the same direction) and the items in $W_{\mathrm{s}}$ are negative in $x$ - and $\psi$-direction (the exciting forces of the two exciters are in opposite directions). It is consistent with the principle of superposition of a linear vibrating system. On the other hand, the item in $W_{\mathrm{c}}$ is negative in $y$-direction and the items in $W_{\mathrm{c}}$ are positive in $x$ - and $\psi$-direction. The effects of $W_{\mathrm{s}}$ and $W_{\mathrm{c}}$ on the load torques and the moments of inertia of two exciters also depend on the phase difference between two exciters. According to the expressions of $W_{\mathrm{s} 0}$ and $W_{\mathrm{c} 0}$ [23], the items $m_{i} \sin \gamma_{x} / m_{x}^{\prime}$, $m_{i} \sin \gamma_{y} / m_{y}^{\prime}$ and $m_{i} l_{0}^{2} \sin \gamma_{\psi} / J^{\prime}$ are called the coefficients of sine effect of phase angle for exciter $i$ in $x-, y$ - and $\psi$-directions (simplified as the coefficient of sine effect), respectively; and the items $m_{i} \cos \gamma_{x} / m_{x}^{\prime}, m_{i} \cos \gamma_{y} / m_{y}^{\prime}$ and $m_{i} l_{0}^{2} \cos \gamma_{\psi} / J^{\prime}$ are called that of cosine effect of phase angle for exciter $i, i=1,2$ (simplified as the coefficient of cosine effect). Hence, $m_{1} W_{\mathrm{s} 0}$ and $m_{2} W_{\mathrm{s} 0}$ are called the coefficients of sine effect of exciter 1 and $2, m_{1} W_{\mathrm{c} 0}$ and $m_{2} W_{\mathrm{c} 0}$ the coefficients of cosine effect of exciters 1 and 2, respectively. $m_{\mathrm{c}} W_{\mathrm{s}}$ and $m_{\mathrm{c}} W_{\mathrm{c}}$ are called the coefficients of coupling sine and cosine effect of two exciters, respectively.

From Eq. (1) and (2), it can be seen that $m_{\mathrm{c}} W_{\mathrm{s}}$ and $m_{\mathrm{c}} W_{\mathrm{c}}$ influence both the load torques and moments of inertia of two exciters due to the occurrence of phase difference between the two exciters. While $m_{1} W_{\mathrm{s} 0}$ and $m_{2} W_{\mathrm{s} 0}$ have only an effect on the torque of its motor, respectively; and $m_{1} W_{\mathrm{c} 0}$ and $m_{2} W_{\mathrm{c} 0}$ have only an effect on the moment of inertia of its exciter, respectively.

In Eq. (1), the load torque of each motor consists of three items. The first is equal to the product of the kinetic energy, $m_{i} r^{2} \omega_{\mathrm{m} 0}^{2} / 2$, and the coefficient of sine effect of its exciter, $m_{i} W_{\mathrm{s} 0}$, which is called the torque of sine effect of the exciter; the second is the product of the coupling kinetic energy, $m_{\mathrm{c}} r \omega_{\mathrm{m} 0}^{2} / 2$, the efficient of sine coupling effect, $m_{\mathrm{c}} W_{\mathrm{s}}$, and the cosine of phase difference of two exciters, $\cos 2 \alpha$, which describes the torque of coupling sine effect; and the last is the product of the coupling kinetic energy, $m_{\mathrm{c}} r \omega_{\mathrm{m} 0}^{2} / 2$, the coefficient of cosine coupling effect, $m_{\mathrm{c}} W_{\mathrm{c}}$, and the sine of phase difference of two exciters, $\sin 2 \alpha$, which expresses the torque of coupling cosine effect. The last two items stem from the coupling effect of two exciters; and their values for two motors are same. However, the load torques stemming from the coupling sine effect for two motors have the same sign, while that stemming from the cosine coupling effect (torque of frequency capture) have the reverse sign, i.e., it is the load torque for the motor with the leading phase and the driving torque for the other with the lagging phase. When $W_{\mathrm{c}}>0$, the stable phase difference $2 \alpha^{*}$ is in the interval of $(-\pi / 2, \pi / 2)$; otherwise, $2 \alpha^{*} \in(\pi / 2,3 \pi / 2)$. These facts demonstrate that the frequency capture stems from the action of the coupling cosine effect of two exciters on the load torques of two motors. The motions of the system excited by two exciters in the same direction makes their phase difference close to $\pi$; and that in opposite directions makes their phase difference close to 0 .

In Eq. (2), there are also three items. The first $4 J_{01}^{\prime} J_{02}^{\prime}$ is four times product of the relative moments of inertia of two exciters; the last two items result from the coupling sine and cosine effects of two exciters, respectively. Eq. (3) demonstrates that the relative moments of inertia of the two exciters reduce due to their cosine effect of the phase angle; and the amount of reduction of each exciter is equal to the product of its moment of inertia and its coefficient of the cosine effect. It decreases the stability of synchronous operation. The coupling cosine effect also decreases the stability of the synchronous operation and the sine coupling effect increases the stability of the synchronous operation. The bigger the damping constants of the system are, the stronger the stability of the system is.

\section{Numerical approach}

\subsection{Calculation of capture angular velocity and the phase difference}

Equations of Frequency Capture is $\mathbf{A} \dot{\varepsilon}=\mathbf{B} \varepsilon+\mathbf{u}$, while $\mathbf{u}=\mathbf{0}$ is the necessary condition of implementing frequency capture of the considered system. Rearranging $u_{1}=0$ and $u_{2}=0$ [23], we have

$$
\begin{aligned}
& 2 \bar{\alpha}=\sin ^{-1} \frac{T_{\mathrm{e} 2}\left(s_{0}\right)-T_{\mathrm{e} 2}\left(s_{0}\right)-P_{\mathrm{m}} s_{0}^{2}\left(1-\eta^{2}\right) W_{s 0}-\frac{\Delta f s_{0} \omega_{\mathrm{s}}}{n_{\mathrm{p}}}}{2 P_{\mathrm{m}} s_{0}^{2} \eta W_{\mathrm{c}}} \\
& F\left(\bar{\alpha}, s_{0}\right)=T_{\mathrm{e} 2}\left(s_{0}\right)+T_{\mathrm{e} 2}\left(s_{0}\right)-\left(f_{\mathrm{d} 1}+f_{\mathrm{d} 2}\right) \omega_{s} s_{0}-P_{\mathrm{m}} s_{0}^{2}\left[\left(1+\eta^{2}\right) W_{s 0}-2 \eta W_{s} \cos 2 \bar{\alpha}\right]
\end{aligned}
$$


where $\Delta f=f_{\mathrm{d} 1}-f_{\mathrm{d} 2}$ and $P_{\mathrm{m}}=\frac{1}{2} m_{0}^{2} r^{2} \omega_{\mathrm{s}}^{2}$. To maintain the continuous operation of two motors, the angular velocity of the synchronous operation must be less than their rated ones, i.e., the slip of the synchronous operation of two motors must be less than the smaller one of their rated slips. If the rated slips of the two motors assumed to be $s_{\mathrm{e} 1}$ and $s_{\mathrm{e} 2}$, respectively, the slip of the synchronous operation $s_{0}$ satisfies

$$
0<s_{0} \leq s_{\mathrm{e}}\left(\min \left\{s_{\mathrm{e} 1}, s_{\mathrm{e} 2}\right\}\right)
$$

$F\left(\bar{\alpha}, s_{0}\right)$ in Eq. (5) is a function of $\alpha$, and satisfies

$$
F(\bar{\alpha}, 0)<0 \text { and } F\left(\bar{\alpha}, s_{\mathrm{e}}\right) \geqslant 0
$$

Because $F\left(\bar{\alpha}, s_{0}\right)$ is the monotonous increasing function of $s_{0}$, it has a unique root in the interval of $\left[0, s_{\mathrm{e}}\right]$. The method of bisection [16] is employed to solve the roots of $\bar{\alpha}$ and $s_{0}$. The steps of this algorithm are summarized as follows

Step 1: Input the parameters of the system. They are the structural parameters of the vibrating system, and the parameters of the two motors, the eccentric radius and the masses of two eccentric lumps and a tolerance $\delta$.

Step 2: Calculate $s_{\mathrm{e}}$.

Step 3: Let $s_{0}=s_{\mathrm{e}}$ and calculate the torque of frequency capture, $T_{\text {Captrue, }}$, and the residual electromagnetic torques of two motors, $T_{\text {Diffenrence }}$ [23], respectively.

Step 4: Compare $T_{\text {Captrue }}$ with $T_{\text {Diffenrence. If }} T_{\text {Captrue }}$ is less than $T_{\text {Diffenrence, }}$, the program is terminated because it is impossible to implement the frequency capture; otherwise, go to Step 5.

Step 5: Calculate $2 \bar{\alpha}$ by using Eq. (4).

Step 6: Insert $2 \bar{\alpha}$ into Eq. (5) to find the value of $F\left(\bar{\alpha}, s_{0}\right)$.

Step 7: Compare $F\left(\bar{\alpha}, s_{0}\right)$ with zero. If $F\left(\bar{\alpha}, s_{0}\right)<0$, the program is terminated because the power of two motors is not enough to drive the vibrating system; otherwise, go to Step 8.

Step 8: Let $a=0$ and $b=s_{\mathrm{e}}$.

Step 9: Let $s_{0}=(a+b) / 2$, i.e., $s_{0}$ is assumed to be the midpoint of $[a, b]$.

Step 10: Calculate $2 \bar{\alpha}$ by using Eq. (4).

Step 11: Insert $2 \bar{\alpha}$ into Eq. (5) to find the value of $F\left(\bar{\alpha}, s_{0}\right)$.

Step 12: Compare $\left|F\left(\bar{\alpha}, s_{0}\right)\right|$ with the positive tolerance $\delta$. If $\left|F\left(\alpha, s_{0}\right)\right|<\delta$, the program is terminated with the solved $2 \alpha^{*}$ and $\omega_{\mathrm{m} 0}^{*}=\left(1-s_{0}\right) \omega_{\mathrm{s}}$. Otherwise, go step 13 .

Step 13: Compare $F\left(\bar{\alpha}, s_{0}\right)$ with zero, if $F\left(\bar{\alpha}, s_{0}\right)<0$, let $a=s_{0}$; otherwise, let $b=s_{0}$, then return Step 9 .

\subsection{Calculation of stability domain}

The characteristic equation for the eigenvalue of equations of frequency capture is rewritten as the following [23]

$$
\lambda^{3}+c_{1} \lambda^{2}+c_{2} \lambda+c_{3}=0
$$

By introducing the following non-dimensional parameters:

$$
\begin{aligned}
& \mu_{1}=1-\frac{m_{0} W_{\mathrm{c} 0}}{2}, \quad \mu_{2}=\eta\left(1-\eta \frac{m_{0} W_{\mathrm{c} 0}}{2}\right), \\
& \kappa_{1}=\frac{f_{\mathrm{d} 1}}{m_{0} r_{0}^{2} \omega_{\mathrm{m} 0}^{*}}+\frac{k_{\mathrm{e} 1}}{m_{0} r_{0}^{2} \omega_{\mathrm{m} 0}^{* 2}}, \\
& \kappa_{2}=\frac{f_{\mathrm{d} 2}}{m_{0} r_{0}^{2} \omega_{\mathrm{m} 0}^{*}}+\frac{k_{\mathrm{e} 2}}{m_{0} r_{0}^{2} \omega_{\mathrm{m} 0}^{* 2}},
\end{aligned}
$$

into the expressions of $c_{1}, c_{2}$ and $c_{3}$ [23], the coefficients of Eq. (8) can be expressed as

$$
c_{1}=\frac{4 \omega_{\mathrm{m} 0}^{*} H_{1}}{H_{0}}, c_{2}=\frac{2 \omega_{m 0}^{* 2} H_{2}}{H_{0}}, c_{3}=\frac{2 \omega_{m 0}^{* 3} H_{3}}{H_{0}}
$$

where, 


$$
\begin{aligned}
& H_{0}=4 \mu_{1} \mu_{2}+\eta^{2} m_{0}^{2} W_{\mathrm{s}}^{2} \sin ^{2} 2 \alpha^{*}-\eta^{2} m_{0}^{2} W_{\mathrm{c}}^{2} \cos ^{2} 2 \alpha^{*} \\
& H_{1}=\mu_{1} \kappa_{2}+\mu_{2} \kappa_{1} \\
& H_{2}=4 \kappa_{1} \kappa_{2}+\eta^{2} m_{0}^{2} W_{\mathrm{c}}^{2}\left(1+\cos ^{2} 2 \alpha^{*}\right)+2\left(\mu_{1}+\mu_{2}\right) \eta m_{0} W_{\mathrm{c}}^{2} \cos 2 \alpha^{*} \\
& H_{3}=\left(\kappa_{1}+\kappa_{2}\right) \eta m_{0} W_{\mathrm{c}} \cos 2 \alpha^{*}
\end{aligned}
$$

$H_{0}, H_{1}, H_{2}, H 3, \mu_{1}$ and $\mu_{2}$ are the dimensionless parameters of the system, in which $\mu_{1}$ and $\mu_{2}$ are the dimensionless relative moment of inertia of two exciters, respectively; and $\kappa_{1}$ and $\kappa_{2}$ are called the non-dimensionless coefficients of velocity stiffness of two motors, respectively.

The equivalent rotating radius of the vibrating system about its centriod is expressed as

$$
l_{\mathrm{e}}=\sqrt{\frac{J+\left(m_{1}+m_{01}\right) l_{0}^{2}+\left(m_{2}+m_{02}\right) l_{0}^{2}}{\left(m+m_{1}+m_{01}+m_{2}+m_{02}\right)}}
$$

where $J$ is the moment of inertia of the machine body rotating about its centroid; $m$ the mass of the vibrating body; $m_{01}$ and $m_{02}$ the masses of motors 1 and 2 .

Then, $m_{0} W_{s 0}, m_{0} W_{s}, m_{0} W_{\mathrm{c} 0}$ and $m_{0} W_{\mathrm{c}}$ can be expressed as

$$
\begin{aligned}
& m_{0} W_{\mathrm{c} 0}=r_{\mathrm{m}}\left(\frac{\cos \gamma_{x}}{1-\omega_{\mathrm{nx}}^{2} / \omega_{\mathrm{m} 0}^{* 2}}+\frac{\cos \gamma_{y}}{1-\omega_{\mathrm{ny}}^{2} / \omega_{\mathrm{m} 0}^{* 2}}+\frac{r_{l}^{2} \cos \gamma_{\psi}}{1-\omega_{\mathrm{n} \psi}^{2} / \omega_{\mathrm{m} 0}^{* 2}}\right) \\
& m_{0} W_{\mathrm{c}}=r_{\mathrm{m}}\left(\frac{\cos \gamma_{x}}{1-\omega_{\mathrm{nx}}^{2} / \omega_{\mathrm{m} 0}^{* 2}}-\frac{\cos \gamma_{y}}{1-\omega_{\mathrm{ny}}^{2} / \omega_{\mathrm{m} 0}^{* 2}}+\frac{r_{l}^{2} \cos \gamma_{\psi}}{1-\omega_{\mathrm{n} \psi}^{2} / \omega_{\mathrm{m} 0}^{* 2}}\right) \\
& m_{0} W_{s 0}=r_{\mathrm{m}}\left(\frac{\sin \gamma_{x}}{1-\omega_{\mathrm{nx}}^{2} / \omega_{\mathrm{m} 0}^{* 2}}+\frac{\sin \gamma_{y}}{1-\omega_{\mathrm{ny}}^{2} / \omega_{\mathrm{m} 0}^{* 2}}+\frac{r_{l}^{2} \sin \gamma_{\psi}}{1-\omega_{\mathrm{n} \psi}^{2} / \omega_{\mathrm{m} 0}^{* 2}}\right) \\
& m_{0} W_{s}=r_{\mathrm{m}}\left(-\frac{\sin \gamma_{x}}{1-\omega_{\mathrm{nx}}^{2} / \omega_{\mathrm{m} 0}^{2}}+\frac{\sin \gamma_{y}}{1-\omega_{\mathrm{ny}}^{2} / \omega_{\mathrm{m} 0}^{2}}-\frac{r_{l}^{2} \sin \gamma_{\psi}}{1-\omega_{\mathrm{n} \psi}^{2} / \omega_{\mathrm{m} 0}^{2}}\right)
\end{aligned}
$$

where $r_{\mathrm{m}}=m_{0} / M$ denotes the ratio of mass of lump 1 (assuming $m_{1} \geqslant m_{2}$ ) to that of the machine body and $r_{l}=l_{0} / l_{\mathrm{e}}$ denotes the ratio of the distance between an exciter and the centroid of the machine body to the equivalent radius of the machine body.

When $H_{0}<0$, the synchronous operation is unstable [23]. Therefore, the stability condition of the system can be expressed as follows

$$
H_{0}>0, \quad H_{1}>0, \quad 4 H_{1} H_{2}-H_{0} H_{3}>0, \quad H_{3}>0
$$

\subsection{Numerical simulation}

Rearranging the equations of motion of the vibrating system [23], we have

$$
\mathbf{Y}=\mathbf{G F}
$$

where $\mathbf{Y}=\left\{\begin{array}{llllll}x & y & \psi & \varphi_{1} & \varphi_{2}\end{array}\right\} ; \mathbf{G}$ and $\mathbf{F}$ are expressed as follows

$$
\begin{aligned}
& \mathbf{G}=\left[\begin{array}{ccccc}
M & 0 & 0 & -m_{1} r \sin \varphi_{1} & m_{2} r \sin \varphi_{2} \\
0 & M & 0 & m_{1} r \cos \varphi_{1} & m_{2} r \cos \varphi_{2} \\
0 & 0 & J & -m_{1} r l_{0} \cos \left(\varphi_{1}+\beta\right) & m_{2} r l_{0} \cos \left(\varphi_{2}+\beta\right) \\
-m_{1} r \sin \varphi_{1} & m_{1} r \cos \varphi_{1}-m_{1} r l_{0} \cos \left(\varphi_{1}+\beta\right) & J_{01} & 0 \\
m_{2} r \sin \varphi_{2} & m_{2} r \cos \varphi_{2} & m_{2} r l_{0} \cos \left(\varphi_{2}+\beta\right) & 0 & J_{02}
\end{array}\right]^{-1} \\
&-k_{x} x-f_{x} \dot{x}+m_{1} r \dot{\varphi}_{1}^{2} \cos \varphi_{1}-m_{2} r \dot{\varphi}_{2}^{2} \cos \varphi_{2} \\
&-k_{y} y-f_{y} \dot{y}+m_{1} r \dot{\varphi}_{1}^{2} \sin \varphi_{1}+m_{2} r \dot{\varphi}_{2}^{2} \sin \varphi_{2} \\
& \mathbf{F}=\left\{\begin{array}{c}
\left.T_{\mathrm{e} 1}-f_{\mathrm{d} 1} \dot{\varphi}_{1}+\beta\right)+m_{2} r l_{0} \dot{\varphi}_{2}^{2} \sin \left(\varphi_{2}+\beta\right) \\
T_{\mathrm{e} 2}-f_{\mathrm{d} 2} \varphi_{2}
\end{array}\right\}
\end{aligned}
$$


Choosing the stator current and the rotor flux as the state variables and the stator voltage as input variables, the state equation of an induction motor with the $d-q$ coordinates fixed in the stator (stator reference frame $(\alpha, \beta)$ ) can be expressed as [14]

$$
\left\{\begin{array}{l}
\mathbf{i}_{\mathrm{s}} \\
\phi_{\mathrm{r}}
\end{array}\right\}=\left[\begin{array}{ll}
\rho_{11} & \rho_{12} \\
\rho_{21} & \rho_{22}
\end{array}\right]\left\{\begin{array}{l}
\mathbf{i}_{\mathrm{s}} \\
\phi_{\mathrm{r}}
\end{array}\right\}+\left[\begin{array}{l}
\mathbf{E}_{1} \\
\mathbf{0}
\end{array}\right] \mathbf{u}_{\mathrm{s}}
$$

where

$$
\begin{aligned}
& \mathbf{i}_{\mathrm{s}}=\left\{\begin{array}{ll}
i_{\alpha \mathrm{s}} & i_{\beta \mathrm{s}}
\end{array}\right\}^{\mathrm{T}}, \phi_{\mathrm{r}}=\left\{\begin{array}{ll}
\phi_{\alpha \mathrm{r}} & \phi_{\beta \mathrm{r}}
\end{array}\right\}^{\mathrm{T}}, \\
& \rho_{11}=-\left[R_{s} /\left(\sigma L_{\mathrm{s}}\right)-(1-\sigma) /\left(\sigma \tau_{\mathrm{r}}\right)\right] \mathbf{I}=\rho_{\mathrm{r} 11} \mathbf{I}, \\
& \rho_{21}=\left(L_{\mathrm{m}} / \tau_{\mathrm{r}}\right) \mathbf{I}=\rho_{\mathrm{r} 21} \mathbf{I} \\
& \rho_{12}=L_{\mathrm{m}} /\left(\sigma L_{\mathrm{s}} L_{\mathrm{r}}\right)\left[\left(1 / \tau_{\mathrm{r}}\right) \mathbf{I}-\omega_{\mathrm{r}} \mathbf{J}\right]=\rho_{\mathrm{r} 12} \mathbf{I}-\rho_{\mathrm{i} 12} \mathbf{J} \\
& \rho_{22}=-\left(1 / \tau_{\mathrm{r}}\right) \mathbf{I}+\omega_{\mathrm{r}} \mathbf{J}=\rho_{\mathrm{r} 22} \mathbf{I}+\rho_{\mathrm{i} 22} \mathbf{J} \\
& \mathbf{u}_{\mathrm{s}}=\left\{\begin{array}{ll}
u_{\alpha s} & u_{\beta s}
\end{array}\right\}^{\mathrm{T}} \\
& \mathbf{E}_{1}=1 /\left(\sigma L_{\mathrm{s}}\right) \mathbf{I}=e_{1} \mathbf{I} \\
& \mathbf{I}=\left[\begin{array}{ll}
1 & 0 \\
0 & 1
\end{array}\right], \quad \mathbf{J}=\left[\begin{array}{ll}
0 & -1 \\
1 & 0
\end{array}\right]
\end{aligned}
$$

Assuming $x_{1}=x, x_{2}=y, x_{3}=\psi, x_{4}=\varphi_{1}, x_{5}=\varphi_{2}, x_{6}=i_{\alpha s 1}, x_{7}=i_{\beta s 1}, x_{8}=\phi_{\alpha \mathrm{r} 1}, x_{9}=\phi_{\beta \mathrm{r} 1}, x_{10}=i_{\alpha \mathrm{s} 2}$, $x_{11}=i_{\beta \mathrm{s} 2}, x_{12}=\phi_{\alpha \mathrm{r} 2}, x_{13}=\phi_{\beta \mathrm{r} 2}, x_{14}=\dot{x}, x_{15}=\dot{y}, x_{16}=\dot{\psi}, x_{17}=\dot{\varphi}_{1}, x_{18}=\dot{\varphi}_{2}$, and substituting them into Eqs (12) and (15), we obtain the equivalent first order equations of the considered system.

$$
\dot{\mathbf{X}}=\mathbf{W}\left(\mathbf{X}, \mathbf{u}_{s} 1, \mathrm{u}_{\mathrm{s} 2}, \mathbf{F}\right)
$$

where $\mathbf{u}_{\mathrm{s} 1}=\left\{\begin{array}{ll}u_{\alpha \mathrm{s} 1} & u_{\beta \mathrm{s} 1}\end{array}\right\}^{\mathrm{T}}$ and $\mathbf{u}_{\mathrm{s} 2}=\left\{\begin{array}{ll}u_{\alpha \mathrm{s} 2} & u_{\beta \mathrm{s} 2}\end{array}\right\}^{\mathrm{T}}$ are input voltages of stator of motors 1 and 2 . $\mathbf{W}=$ $\left\{\begin{array}{llll}w_{1} & w_{2} & \cdots & w_{18}\end{array}\right\}^{\mathrm{T}}$ is the nonlinear function of $\mathbf{X}$, as shown in Eqs (12) and (15).

The integration of Eq. (16) can be carried out using a Runge-Kutta routine with adaptive stepsize control [16].

\section{Results and discussion}

In this section, an example is presented to investigate the main results of theoretical analysis [23]. To verify the feature of frequency capture of the considered system, two motors are assumed to be different and their parameters are listed in Appendix.

To determine the stability domain that satisfies Inequation (11), $r_{\mathrm{m}}$ is fixed to solve $r_{l}$ that satisfies $H_{0}=0$, $H_{1}=0,4 H_{1} H_{2}-H_{0} H_{3}=0$ and $H_{3}=0$, respectively. The power of the system should be less than or equal to the sum of power of two motors. The radius of the eccentric lumps is adjusted to meet the requirement of the power of two motors. If the phase difference between the two exciters satisfies $\sin 2 \alpha^{*} \approx 0$, the sum of external load torques of two motors, denoted by $T_{\mathrm{SL}}$, can be approximately simplified as the follows

$$
T_{\mathrm{SL}} \approx \frac{1}{2} m_{0}^{2} r^{2} \omega_{\mathrm{m} 0}^{2}\left[\left(1+\eta^{2}\right) W_{\mathrm{s} 0}+2 \eta W_{\mathrm{s}}\right]
$$

When two motors operate at the angular velocity $\omega_{\mathrm{me}}=\left(1-s_{\mathrm{e}}\right) \omega_{\mathrm{s}} / n_{\mathrm{p}}$, the electromagnetic torques of motors 1 and 2 are denoted by $T_{\mathrm{e} 01}$ and $T_{\mathrm{e} 02}$. Hence, the eccentric radius of two exciters is calculated by

$$
r=\frac{\sqrt{\chi}}{m_{0} \omega_{\mathrm{me}}} \sqrt{\frac{2\left(T_{\mathrm{e} 01}+T_{\mathrm{e} 02}\right)}{\left(1+\eta^{2}\right) W_{\mathrm{s} 0}-2 \eta W_{\mathrm{s}}}}
$$

where $\chi=0.95 \sim 1.0$ denotes the coefficient of power use of the two motors in the vibrating system. 
$r_{\mathrm{m}}$ is assumed in the range of 0.01 to 0.2 and $\chi=0.95$. To ensure the torque of frequency capture high enough to overcome the difference between the residual torques of the two motors, the structural parameter of the vibrating system must satisfy $\left|H_{3}\right|>0$. Considering $c_{3}>0$, we can adjust $2 \alpha^{*}$ in the intervals of $(-\pi / 2, \pi / 2)$ and $(\pi / 2,3 \pi / 2)$ to ensure that the sign of $H_{3}$ is the same as that of $H_{0}$. Therefore, the stability domain of synchronous operation of two motors depends on the signs of $H_{0}, H_{1}$ and $H=4 H_{1} H_{2}-H_{0} H_{3}$.

$H$ is a function of higher order of the variables $r_{\mathrm{m}}$ and $r_{l}$. Figure 1(a) shows the relationships between $H$ and $r_{l}$ when $r_{\mathrm{m}}$ is $0.03,0.05,0.06,0.1$ and 0.2 , respectively. It should be noticed that there is only one zero point in each curve (the corresponding value of $r_{l}$ is denoted by $r_{l 0}$ ). The less $r_{\mathrm{m}}$ is, the greater $r_{l 0}$. According to the stability condition of Inequation (11), $r_{l}<r_{l 0}$ satisfies $c_{1} c_{2}>c_{3}$. Figure 1(b) shows the distribution diagram of zero points of $H_{0}\left(r_{\mathrm{m}}, r_{l}\right)$ in $r_{\mathrm{m}} r_{l}$-plane. $H_{0}\left(r_{m}, r_{l}\right)$ is a second order function of the parameters $\left(r_{\mathrm{m}}, r_{l}^{2}\right)$, so there are two branch curves $\left(H_{01}\right.$ and $\left.H_{02}\right)$ to satisfy $H_{0}\left(r_{\mathrm{m}}, r_{l}\right)=0 . H_{0}\left(r_{\mathrm{m}}, r_{l}\right)$ is less than zero in the region between two branches $H_{01}$ and $H_{02}$, and $H_{0}\left(r_{\mathrm{m}}, r_{l}\right)>0$ beyond this region. Figure 1(c) shows the stability regions (diagonally hatched) of synchronous operation of two motors in the terms of the parameter $\left(r_{m}, r_{l}\right)$ and the distributions of $H_{1}=0$ and $H=0$. It can be seen that $H_{0}$ firstly becomes negative, then $H$ followed by $H_{1}$. Therefore, $H_{0}>0$, $\mu_{1}>0$ and $\mu_{2}>0$ are the conditions of stability of synchronous operation. This result is consistent with theoretical results [23]. Figure 1(d) shows the relationship between the torque of frequency capture and $r_{l}$, which is independent on $r_{\mathrm{m}}$. When $r_{l}$ varies initially from its minimum, the torque of frequency capture is approximately proportional to $r_{l}$. However, with the increase of $r_{l}$, the torque of frequency capture is gradually close to a limit value. The reason for this phenomenon is that the mass difference between two exciters leads to the swing vibration of the machine body that increases the damping energy consumption of the swing vibration. The greater $r_{l}$ is, the bigger the damping energy consumption of the swing vibration. This results in a less value of the eccentric radius, $r$, as shown in Eq. (18).

Tables 1 and 2 list the critical $r_{l}$-values at which the synchronous operation begins to un-stabilize when the mass ratio $(\eta)$ of the two exciters and the ratios of exciting frequency to natural frequencies of the vibrating system vary. From Tables 1 and 2, it can be seen that the difference in the ratios of exciting frequency to natural frequencies has very limited effect on the stability of synchronous operation in a non-resonant vibrating system. With the comparison of Fig. 1 and Tables 1 and 2, $H_{0}$ always firstly passes the zero line and becomes negative. When $H_{1}$ pass their zero lines, at least one of $\mu_{1}$ and $\mu_{2}$ has become negative, see the expression of $H_{1}$ in Eq. (8).

Figure 2 shows the cosine of phase difference along the curve $H_{0}=0$ in Fig. 1(c) when the system operates at the steady-state. It can be seen that the cosine of phase difference at the critical point of the stability of the synchronous operation is very close to 1 , i.e., $\sin 2 \alpha$ is very close to 0 . Therefore, the coefficient of coupling sine effect on $H_{0}$-value can be ignored. The dimensionless parameter $H_{0}$, which determines the stability of synchronous operation, can be rewritten as

$$
H_{0}=4 \mu_{1} \mu_{2}-m_{0}^{2} \eta^{2} W_{\mathrm{c}}^{2}>0
$$

Equation (19) demonstrates that the stability of the synchronous operation is dependent on the structural parameters of the vibrating system and independent of the parameters of two motors. The parameters of two motors have an effect on implementing the frequency capture. The less the difference in the residual torque of two motors, the easier the realization of frequency capture is. When the magnetic torques of two motors are respectively equal to their load torques, the difference in their residual torques is zero. By designing the suitable mass ratio of two exciters to match electromagnetic torques of two motors, two exciters can operate synchronously at the zero phase difference. If $2 \alpha$ is zero, the nominator of Eq. (4) is zero, thus the condition of implementing frequency capture can expressed as follows [23]

$$
\begin{aligned}
& T_{\mathrm{e} 01}+T_{\mathrm{e} 02}-\frac{1}{2} m_{0}^{2} r^{2} \omega_{\mathrm{m} 0}^{2}\left[\left(1+\eta^{2}\right) W_{\mathrm{s} 0}-2 \eta W_{\mathrm{s}}\right]-\omega_{\mathrm{m} 0}\left(f_{d 1}-f_{\mathrm{d} 2}\right)=0 \\
& T_{\mathrm{e} 01}-T_{\mathrm{e} 02}-\frac{1}{2} m_{0}^{2} r^{2} \omega_{\mathrm{m} 0}^{2}\left(1-\eta^{2}\right) W_{\mathrm{s} 0}-\omega_{\mathrm{m} 0}\left(f_{\mathrm{d} 1}-f_{\mathrm{d} 2}\right)=0
\end{aligned}
$$

Solving Eq. (20) by numerical method to obtain $\omega_{\mathrm{m} 0}$ and $\eta, \eta$ is the optimal match ratio of the masses of two exciters. If $\eta$ satisfies Eq. (20) and $\omega_{\mathrm{m} 0}$ satisfies Eq. (6), the stability domain of synchronous operation reaches its maximum.

Figure 3 shows the results of computer simulation of the vibrating system that the parameters are listed in appendix. When the system starts to operate, the phase of exciter 1 leads that of exciter 2, and the phase difference between 

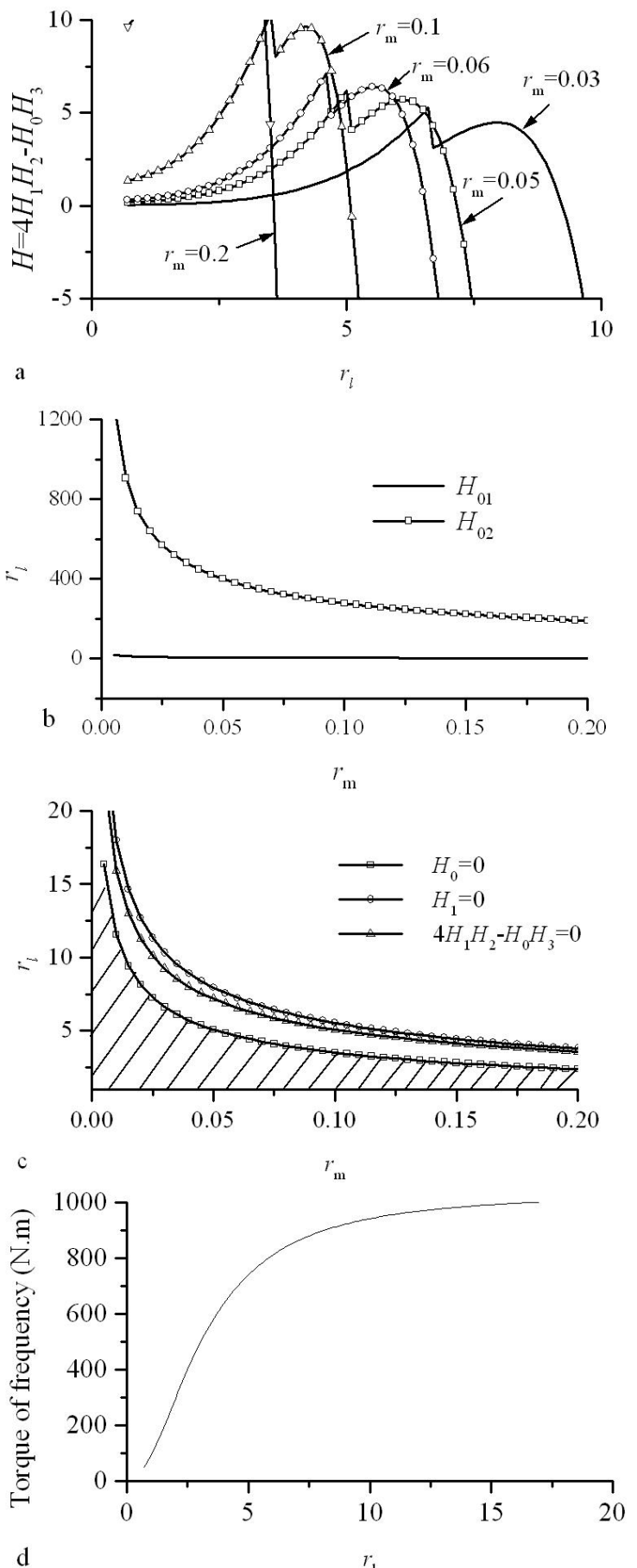

Fig. 1. Numeric results when $\omega_{n x} / \omega_{\mathrm{m} 0}^{*}=\omega_{\mathrm{ny}} / \omega_{\mathrm{m} 0}^{*}=\omega_{\mathrm{n} \psi} / \omega_{\mathrm{m} 0}^{*}=4.5$ and $\eta=0.5$ : a- distribution diagram of $H$ zero value points; b-the distribution diagram of zero point in $r_{\mathrm{m}} r_{1}$-plane; c-the stability domain of synchronous operation; d-torque of frequency capture.

them increases gradually because the electromagnetic torque of motor 1 is greater than that of motor 2 , as shown in Fig. 4(b). When the phase difference is in the interval of $\left(-180^{\circ}, 0^{\circ}\right)$, the torque of frequency capture acts on motor 1 as driving torque, and on motor 2 as load torque, which makes the phase difference increase rapidly and tends to 
Table 1

Table 1 Effect of mass ratio $(\eta)$ of the two exciters on the stability of the synchronous operation

\begin{tabular}{ccccc}
\hline$\eta$ & $r_{\mathrm{m}}$ & $r_{l}\left(H_{0}=0\right)$ & $r_{l}\left(H_{1}=0\right)$ & $r_{l}(H=0)$ \\
\hline 1.0 & 0.01 & 10.00 & 14.15 & 10.33 \\
& 0.1 & 3.02 & 4.26 & 3.66 \\
& 0.2 & 2.01 & 2.85 & 2.64 \\
0.5 & 0.01 & 11.55 & 18.0 & 15.92 \\
& 0.1 & 3.51 & 5.53 & 5.08 \\
& 0.2 & 2.37 & 3.78 & 3.57 \\
0.2 & 0.01 & 12.94 & 19.08 & 18.94 \\
& 0.1 & 3.93 & 5.88 & 5.85 \\
& 0.2 & 2.64 & 4.04 & 4.02 \\
\hline
\end{tabular}

Table 2

Effect of ratio of exciting frequency to natural frequencies of the vibrating system on the stability of the synchronous operation

\begin{tabular}{ccccc}
\hline Ratio of Freq. & $r_{\mathrm{m}}$ & $r_{l}\left(H_{0}=0\right)$ & $r_{l}\left(H_{1}=0\right)$ & $r_{l}(H=0)$ \\
\hline$\lambda_{x}=2.5$, & 0.01 & 10.87 & 16.94 & 15.25 \\
$\lambda_{y}=2.5$, & 0.05 & 3.29 & 5.19 & 4.84 \\
$\lambda_{\psi}=2.5$. & 0.1 & 2.20 & 3.53 & 3.38 \\
$\lambda_{x}=4.5$, & 0.01 & 10.86 & 16.91 & 14.98 \\
$\lambda_{y}=2.5$, & 0.1 & 3.28 & 5.18 & 4.79 \\
$\lambda_{\psi}=4.5$. & 0.2 & 2.20 & 3.52 & 3.35 \\
$\lambda_{x}=2.5$, & 0.01 & 10.87 & 16.95 & 15.24 \\
$\lambda_{y}=4.5$, & 0.1 & 3.29 & 5.19 & 4.83 \\
$\lambda_{\psi}=2.5$. & 0.2 & 2.20 & 3.53 & 3.36 \\
\hline
\end{tabular}

Note: $\lambda_{x}=\omega_{\mathrm{m} 0}^{*} / \omega_{\mathrm{nx}}, \lambda_{y}=\omega_{\mathrm{m} 0}^{*} / \omega_{\mathrm{ny}}, \lambda_{\psi}=\omega_{\mathrm{m} 0}^{*} / \omega_{\mathrm{n} \psi}$

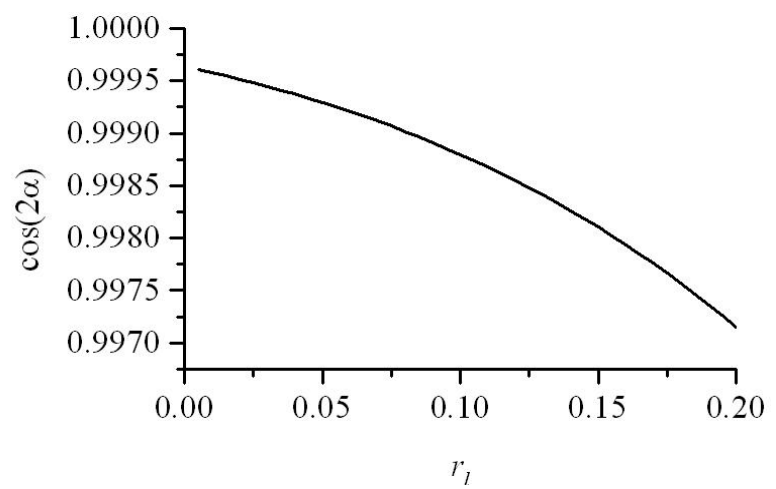

Fig. 2. Cosine of phase difference between the two eccentric rotors along the curve $H_{0}=0$ in Fig. 1(c).

enter in the interval of $\left(0^{\circ}, 180^{\circ}\right)$. During this process, the rotational speed of motor 1 is over its synchronous value $(1033 \mathrm{r} / \mathrm{min})$. When the phase difference enters into the interval of $\left(0^{\circ}, 180^{\circ}\right)$, the torque of frequency capture acts on motor 1 as load torque and on motor 2 as driving torque. The rotational speed of motor 1 decreases rapidly and that of motor 2 increases (the maximum is $1045 \mathrm{r} / \mathrm{min}$ ). Finally, the phase difference reaches $7.9^{\circ}$ and the system operates synchronously.

Because the load torques acting on two motors is periodic, their rotational speeds are also periodic as shown in Fig. 3(a). When $t=2 \mathrm{~s}$, the power supply of motor 2 is cut off, i.e., the electric source is only supply to motor 1. The phase difference increases to $15.7^{\circ}$ and the synchronous operation of two motors continues, which means the vibratory synchronous transmission. When $t=3 \mathrm{~s}$, a $90^{\circ}$ phase disturbance is added to exciter 2 . The system can automatically adjust the phase difference and re-reaches the steady-state of vibratory synchronous transmission. 

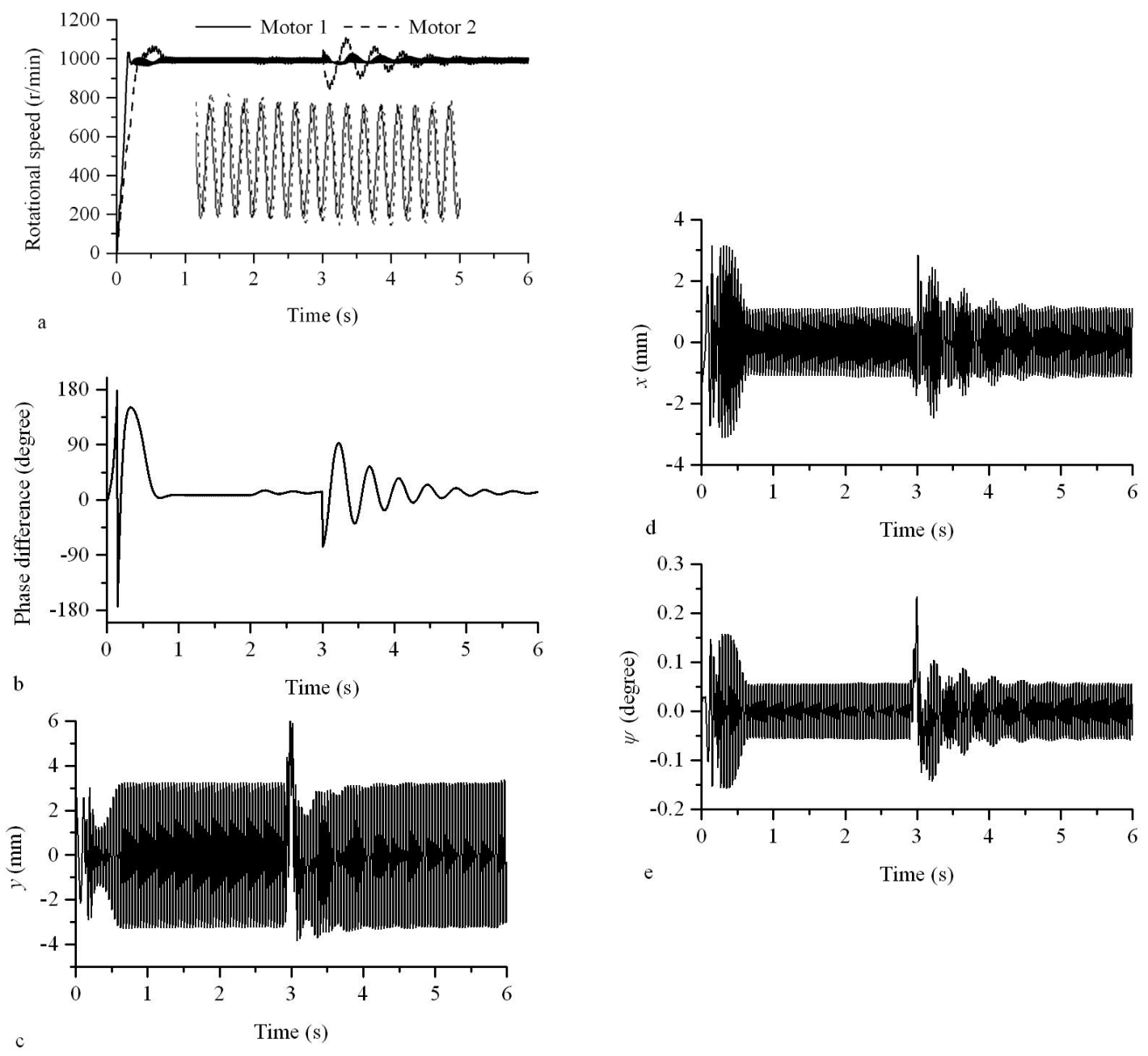

Fig. 3. Results of computer simulation of a vibrating system with two non-identical exciters.

\section{Conclusions}

In the vibrating system with dual-exciters, the vibration of the system excited by two exciters has an effect on the load torques and the moments of inertia of two exciters. The load torque of each motor consists of three items, such as the torque of sine effect, that of coupling sine effect and that of coupling cosine effect. The first item stems from the vibration of the system excited by one exciter on its own motor. The last two items stem from the vibration excited by one exciter on the other motor, which represent the coupling effect of two exciters. The torque of frequency capture stems from the action of coupling cosine effect on two motors. The motions of the system excited by two exciters in the same direction makes their phase difference close to $\pi$ and that in opposite directions makes their phase difference close to 0 . The condition of implementing the frequency capture depends on the parameters of two motors and the coefficient of coupling cosine effect of the two exciters. The matching of the mass ratio of two exciters and the parameters of two motors promotes the implement of frequency capture.

The stability of the synchronous operation is only dependent on the structural parameters of the system, including the mass ratio of two exciters to that of the vibrating system, $r_{\mathrm{m}}$, and the ratio of the distance between one exciter and the centroid of the system to the equivalent radius of the system about its centroid, $r_{l}$. The condition of stability of synchronous operation is that the dimensionless relative moments of inertia of two exciters are all greater than 
zero, and four times of their product is greater than the square of their coefficient of coupling cosine effect. The less $r_{\mathrm{m}}$, the bigger is $r_{l}$. To guarantee the reliable stability of synchronous operation and torque of frequency capture high enough to overcome the difference in the residual toques of two motors, the exciters should be designed to have the greater eccentric radius and the less mass.

When the torque of frequency capture is greater than the bigger one of electromagnetic torques of two motors, the vibratory synchronous transmission can be realized. During the vibratory synchronous transmission, the torque of frequency capture transmits the driving torque of the motor supplied with electric source to the other motor that its power supply is cut off.

\section{Acknowledgment}

We would like to express our appreciation to the key project of the National Science Foundation of China (Grant No: 50535010) and High-tech Research and Development Program of China (Grant No: 2007AA04Z442).

\section{Appendix: Parameters of the vibrating system}

Table A1

Parameters of two induction motors

\begin{tabular}{lll}
\hline Parameters & Motor 1 & Motor 2 \\
\hline Rated power $(\mathrm{kW})$ & 3.7 & 0.75 \\
Poles & 6 & 6 \\
Rated frequency $(\mathrm{Hz})$ & 50 & 50 \\
Rated voltage $(\mathrm{V})$ & 220 & 220 \\
Rated angular velocity $(\mathrm{r} / \mathrm{min})$ & 1780 & 1760 \\
Stator resistance $(\Omega)$ & 0.54 & 3.35 \\
Rotor resistance referred to stator $(\Omega)$ & 0.56 & 3.40 \\
Stator inductance $(\mathrm{H})$ & 0.075 & 0.17067 \\
Rotor inductance referred to stator $(\mathrm{H})$ & 0.075 & 0.17067 \\
Magnetizing inductance $(\mathrm{H})$ & 0.0738 & 0.16373 \\
Damping coefficient of axis $(\mathrm{N} . \mathrm{m} \mathrm{s} / \mathrm{rad})$ & 0.02 & 0.005 \\
\hline
\end{tabular}

Table A2

Parameters of the vibrating system

\begin{tabular}{ll}
\hline Parameter & Value \\
Mass of body of the vibrating system, $M, \mathrm{~kg} ;$ & 1200 \\
Moment of Inertia of about its centriod, $J, \mathrm{~kg} \cdot \mathrm{m}^{2} ;$ & 1058 \\
Mass of eccentric lump 1, $m_{01}, \mathrm{~kg} ;$ & 40 \\
Mass of eccentric lump $2 m_{02}, \mathrm{~kg} ;$ & 20 \\
Spring constant in the direction of $x, k_{x}, \mathrm{~N} / \mathrm{m} ;$ & 630500 \\
Spring constant in the direction of $y, k_{y}, \mathrm{~N} / \mathrm{m} ;$ & 630500 \\
Spring constant in the direction of $\psi, k_{\psi}, \mathrm{N} \cdot \mathrm{m} / \mathrm{rad} ;$ & 556000 \\
Damping constant in the direction of $x, f_{x}, \mathrm{~N} /(\mathrm{m} / \mathrm{s})$ & 3850 \\
Damping constant in the direction of $y, f_{y}, \mathrm{~N} /(\mathrm{m} / \mathrm{s}) ;$ & 3850 \\
Damping constant in the direction of $\psi, f_{\psi}, \mathrm{N} \cdot \mathrm{m} /(\mathrm{rad} / \mathrm{s}) ;$ & 3400 \\
Distance between the lumps and the center of mass, $l_{0}, \mathrm{~m} ;$ & 0.8 \\
Radius of the eccentric lumps, $r, \mathrm{~m}$. & 0.08 \\
\hline
\end{tabular}

\section{References}

[1] J.L. Balthazar, J.L. Palacios and R.B. Felix, Some Comments on the numerical Simulation of Self-Synchronization of Four Non-Ideal Exciters, Applied Mathematics and Computation 164 (2005), 615-625.

[2] I.I. Blekhman, Synchronization of Dynamical Systems, Nauka, Moscow, (in Russian), 1971.

[3] I.I. Blekhman, Synchronization in Science and Technology, ASME Press, New York, 1988. 
[4] I.I. Blekhman, A.L. Fradkov, H. Nijmeijer et al., On Self-Synchronization and Controlled Synchronization, Systems \& Control Letters 31 (1997), 299-305.

[5] I.I. Blekhman, A.L. Fradkov, O.P. Tomchina et al., Self-Synchronization and controlled Synchronization: General Definition and Example Design, Mathematics and Computers in Simulation 58 (2002), 367-384.

[6] J. Chen, Mathematical Model and Speed Adjustment System of Alternating Motors, Defense Press, Beijing, 1989.

[7] K. Czolczynski, P. Perikowski et al., Synchronization of self-excited oscillators suspended on elastic structure, Chaos, Solitons and Fractals 32 (2007), 937-943.

[8] A. Fradkov and B. Andrievsky, Singular Perturbations of Systems Controlled by Energy-Speed-Gradient Method, 43rd IEEE Conference on Decision and Control, December 14-17, Atlantis, Paradise Island, Bahamas, (2004), 3441-3446.

[9] J.F. He, Z.G. Ye, H.Z. Jiang et al., Analysis Based on Joint-Space Model of Parallel Robots, Chinese Journal of Mechanical Engineering 43(6) (2006), 161-165, (in Chinese).

[10] C. Huygens, Horogium Oscilatorium, Paris, France, 1673.

[11] Q.S. Lu, Qualitative Methods and Bifurcations of Ordinary Differential Equations, Press of Beijing University of Aeronautics and Astronautics, Beijing, (in Chinese), 1989.

[12] G.V. Osipov, J. Kurths and C.S. Zhou, Synchronization in Oscillatory Networks, Springer Berlin Heidelberg, 2007.

[13] J. Rayleigh, Theory of Sound, Dover, New York, 1945.

[14] L. Salvaio, S. Stasi and L. Tarchioni, A New EKF-Algorithm for Flux Estimation in Induction Machines, IEEE Transactions on Industrial Electronics 40(5) (1993), 496-501.

[15] A. Teufel, A. Steindl and H. Troger, Synchronization of two flow excited pendula, Communication in Nonlinear Science and Numerical Simulattion 11 (2006), 577-594.

[16] C.F. van Loan, Introduction to Scientific Computing: A Matrix-Vector Approach Using Matlab, China Machine Press, Beijing, 2005.

[17] B. van der Pol, Theory of the Amplitude of Free and Forced Triode Vibration, Radio Rev 1 (1920), 701-710.

[18] B.C. Wen, Y.N. Li, Y.M. Zhang et al., Vibration Utilization Engineering, Science Press, Beijing, (in Chinese), 2005.

[19] B.C. Wen and F.Q. Liu, Theory of Vibrating Machines and Its Applications, Mechanical Press, Beijing, (in Chinese), 1982.

[20] B.C. Wen, C.Y. Zhao, D.P. Su et al., Vibration Synchronization and Controlled Synchronization, Science Press, Beijing, (in Chinese), 2003.

[21] B.C. Wen, Recent Development of Vibration Utilization Engineering, Fronties Mechanical Engineering in China 2(1) (2008), 1-9.

[22] B.C. Wen, Y.N. Li, Q.K. Han et al., Nonlinear Vibration in Engineering, Science Press, Beijing, (in Chinese), 2007.

[23] C.Y. Zhao, R.Z. Wang, T.J. Bai et al., Synchronization of Two Dissimilar Coupled Exciters in an Un-Resonant Vibrating System of Linear Motion, Part I: Theoretical Analysis, Shock and Vibration 16 (2009), 505-515, 

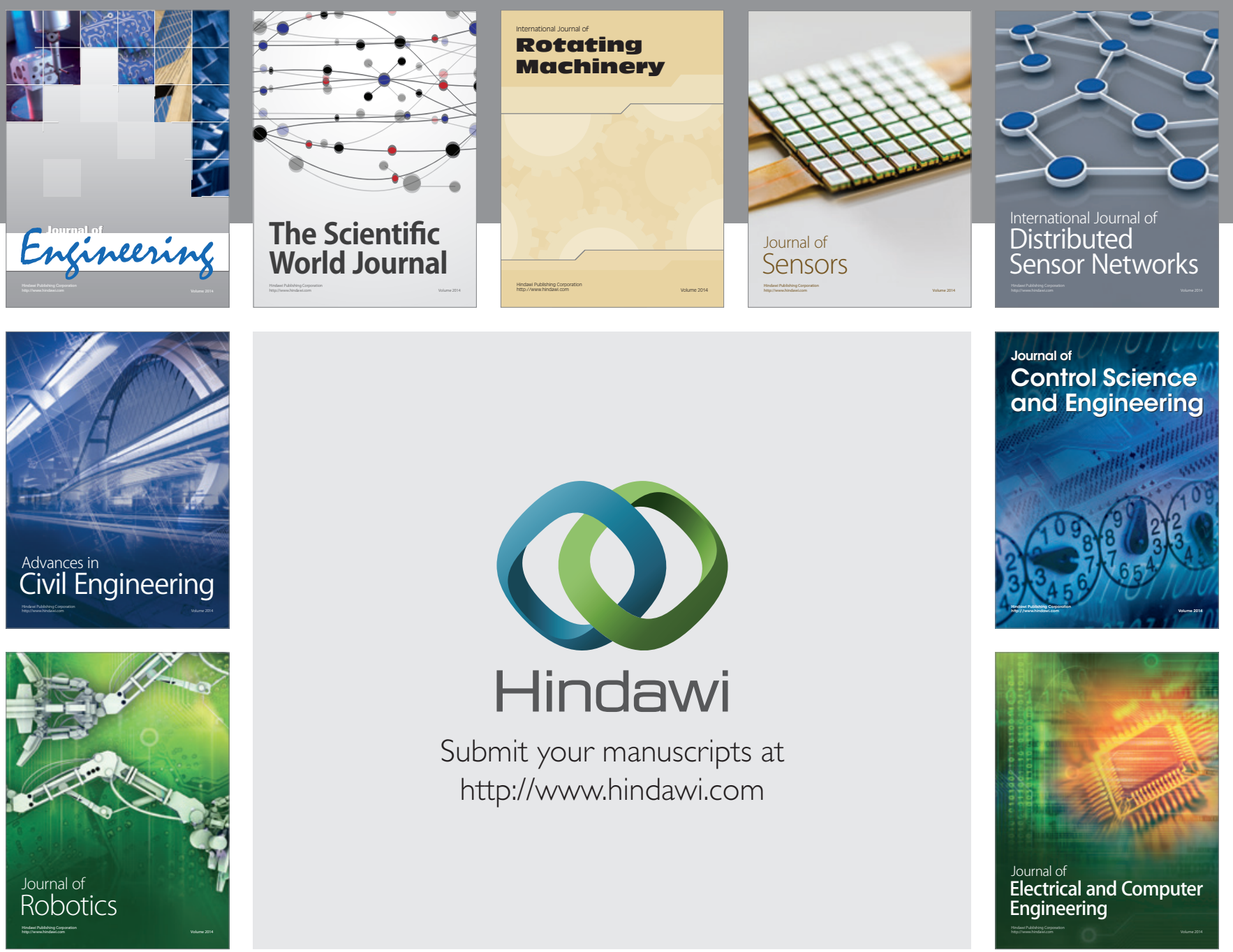

Submit your manuscripts at

http://www.hindawi.com
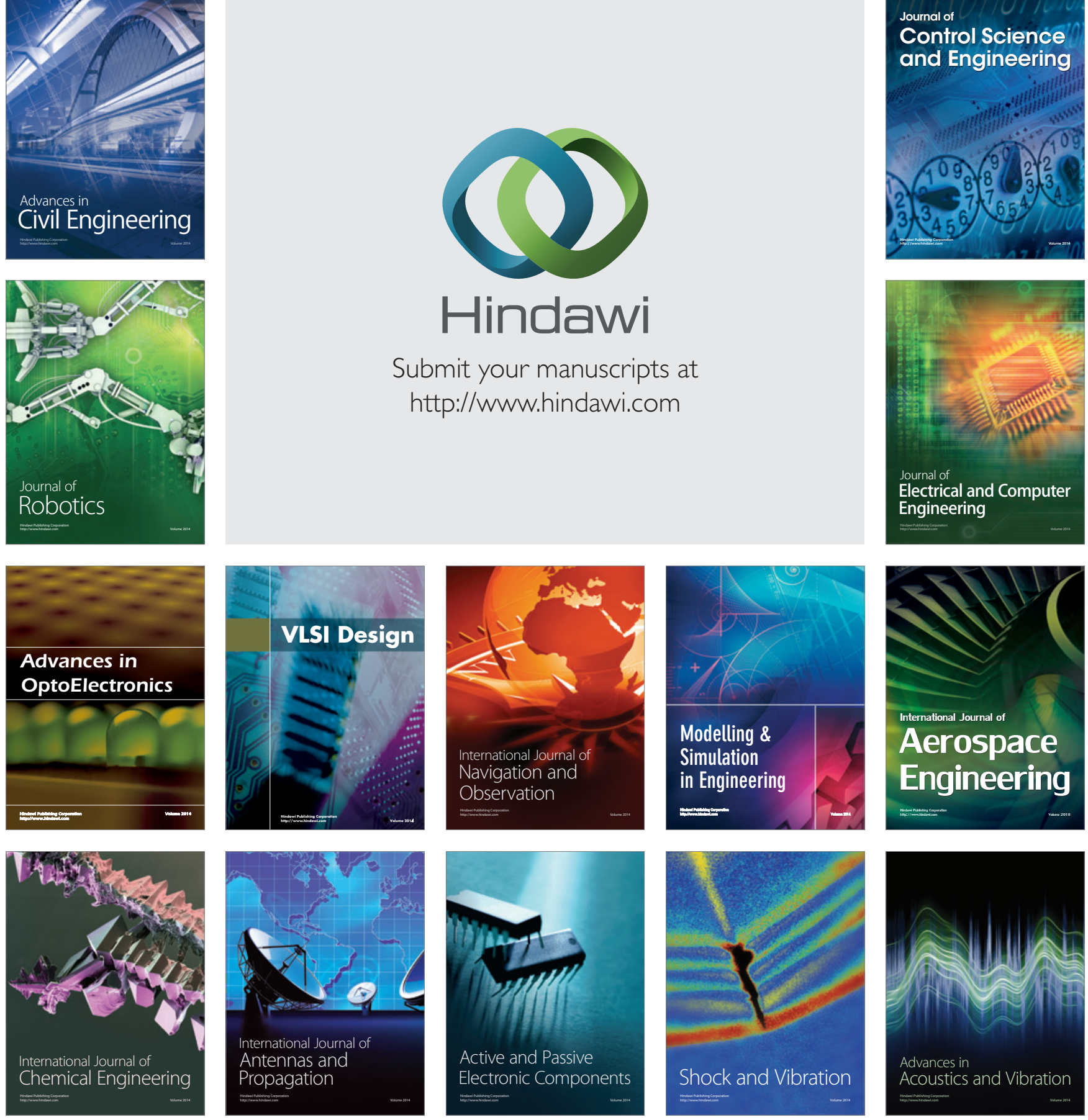